\title{
Study on Buckling Failure Criterion for Compression-shear Sliding Unstable Rock
}

\author{
Jinhao Zhang ${ }^{1, *}$ and Hongkai Chen ${ }^{1}$ \\ ${ }^{1}$ Institute of Geotechnical Engineering, \\ Chongqing Jiaotong University, \\ Chongqing 400074, China \\ E-mail:haohaoandbobo@163.com;
}

\author{
He Wang ${ }^{1}$ and Renjie $\mathrm{Wu}^{1}$ \\ ${ }^{1}$ Institute of Geotechnical Engineering, \\ Chongqing Jiaotong University, \\ Chongqing 400074, China
}

\begin{abstract}
The essence of failure for compression-shear sliding unstable rock belongs to the fracture of dominant fissure. The fracture mechanics model of compression shear type dangerous rock is established by combining rock failure criterion with fracture strength factor; By equivalent stress method, the equivalent stress field of the non - parallel section of the main control surface is obtained; The failure criterion of compression shear type dangerous rock is derived by using the classical D-P failure theory.
\end{abstract}

Keywords-rock and soil mechanics; equivalent stress; fracture criterion; dominant fissure; compression-shear sliding unstable rock

\section{INTRODUCTION}

Unstable rock failure is a serious global mountain disaster problem with sudden, rapid and destructive characteristics. According to incomplete statistics, China's mountainous towns, mines, along the main road along the dangerous, dangerous, security level potential instability of tens of thousands of, only the Three Gorges Reservoir area of Chongqing, there are more than 50 thousand towns. There are more than 8000 times of instability disasters in China every year, and the direct economic loss is about 5 billion, which seriously threatens people's personal and property security. Therefore, the study of the mechanism of instability and failure has become the focus of many scholars. The most representative ones are, Chen Hongkai and so on [1-6] used the fracture mechanics method to establish the joint fracture strength factor computation method of the main control structure plane crack extension direction, combined with the fracture toughness of dangerous rock, the stability calculation method of dangerous rock is established; Liu Changjun et al [7] classify the spatial mass model according to the unstable type of dangerous rock mass, the establishment of rock anchorage calculation method; Yu Mingming et al [8] adopted cantilever beam mechanical model, the stability of dangerous rock mass on high slope is calculated and analyzed quantitatively; Zhang Yongxing et al [9] studied the influence of the tensile stress in the slope and the depth of the cavity development on the formation and destruction of the weathered weathered rock, and proposed a method to predict the controlled failure modes of the weathered weathered rock; Zheng Anxing et al [10] will control fissure analogy to macroscopic crack, using extended finite element method (XFEM), to solve the structural plane propagation process of moving discontinuous problems, and explain under the load of dangerous rock master structure surface fracture behavior; Shi Yucheng and so on [11]according to the different rock dynamic displacement field of prestressed anchor reinforcement loads, the stress field distributions are simulated, reveals the dynamic response and the change rule of prestressed anchor cable in rock under seismic action. based on the theory of fracture mechanics, it is considered that the nature of the failure of the compression shear type dangerous rock (Figure 1) is the fracture of the main control structural plane [12]. The fracture mechanics model of compression shear type dangerous rock is established by combining rock failure criterion with fracture strength factor; Considering the two cases of no crack water pressure stress and fissure water pressure stress in the through section of the main structure of the perilous rock, the equivalent stress field of the non parallel section of the main structure face is obtained by the stress equivalent method; The application of Drucker-Prager criterion, shear sliding type rock failure criterion is deduced. It is of great applicability to explain the failure mechanism of this kind of dangerous rock.

\section{A MECHANICAL MODEL OF COMPRESSION SHEAR SLIDING DANGEROUS ROCK IS ESTABLISHED}

The core problem of the formation and failure mechanism of compressive shear type dangerous rock is the fracture growth of the main controlled structural plane under the combined action of compressive and shear stresses. Therefore, the establishment of mechanical model (Figure 2), the angle ( $\beta$ ) of the main control structure plane in the picture, the main control structure of the load are mainly rock weight $\mathrm{W}$, seismic force (horizontal seismic force $\mathrm{Ph}$ and vertical seismic force $\mathrm{Pv}$ ) and fissure water pressure stress $\mathrm{Pw}$, performance control structure surface compressive stress the shear stress and (Figure 2). Based on the analysis of the model, the model assumptions in plane stress condition, rock masses as with edge crack of the proposed finite template, fracture mechanics model of compressive shear sliding rock. The model takes into account the structure of the main control section through the effect of fissure water pressure stress (figure 3). In Figure 3, $\mathrm{AB}$ as the main control plane; The structure of the $\mathrm{AO}$ as the main control through segment, the length is $\mathrm{a}$; $\mathrm{AO}$ main control structural face through section, length is a; OB non controlled section with main control structure, length is a0; 
The coordinate origin $\mathrm{O}$ point is at the end of the through section; Based on the assumption of plane stress, the force does not change along the thickness, and the main structure of the dangerous rock is subjected to compression, stress and shear stress, and presents the composite fracture of compression shear (I-II).



Figure 1. Picture of compression-shear sliding unstable rock;

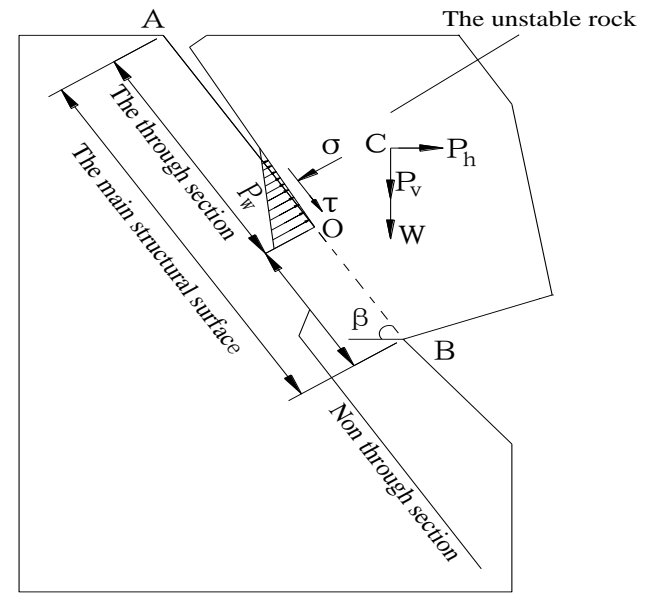

Figure 2. Mechanical model of compression-shear sliding unstable rock

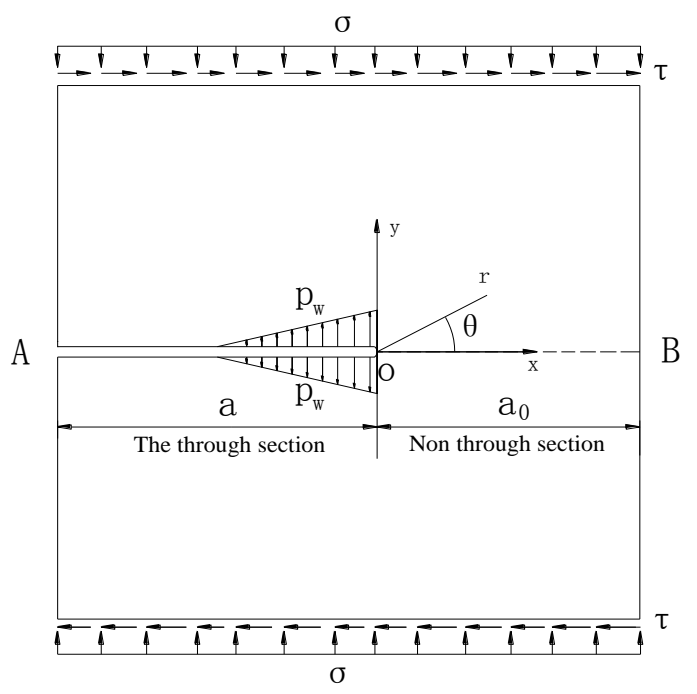

Figure 3. Mechanical model of hydrostatic stress fracture acting connective section;



Figure 4. Stress equivalent model of control fissure

\section{SOLVING THE EQUIVALENT STRESS FIELD AT THE TIP OF THE MAIN CONTROL PLANE}

The stress field at the tip of crack can be obtained by superposition principle of compression shear (I-II) sliding dangerous rock

For the I mode, the stress field at the tip of the crack (when the compressive stress is negative): 


$$
\left\{\begin{array}{l}
\sigma_{x}=-\frac{K_{\mathrm{I}}}{\sqrt{2 \pi r}} \cos \frac{\theta}{2}\left[1-\sin \frac{\theta}{2} \sin \frac{3 \theta}{2}\right] \\
\sigma_{y}=-\frac{K_{\mathrm{I}}}{\sqrt{2 \pi r}} \cos \frac{\theta}{2}\left[1+\sin \frac{\theta}{2} \sin \frac{3 \theta}{2}\right] \\
\sigma_{x y}=-\frac{K_{\mathrm{I}}}{\sqrt{2 \pi r}} \cos \frac{\theta}{2} \sin \frac{\theta}{2} \cos \frac{3 \theta}{2}
\end{array}\right.
$$

For the II type crack tip stress field:

$$
\left\{\begin{array}{l}
\sigma_{x}=-\frac{K_{\amalg}}{\sqrt{2 \pi r}} \sin \frac{\theta}{2}\left[2+\cos \frac{\theta}{2} \cos \frac{3 \theta}{2}\right] \\
\sigma_{y}=\frac{K_{\amalg}}{\sqrt{2 \pi r}} \cos \frac{\theta}{2} \sin \frac{\theta}{2} \cos \frac{3 \theta}{2} \\
\tau_{x y}=\frac{K_{\amalg}}{\sqrt{2 \pi r}} \cos \frac{\theta}{2}\left[1-\sin \frac{\theta}{2} \sin \frac{3 \theta}{2}\right]
\end{array}\right.
$$

The stress fields at the crack tip of I and II modes at the end of the through section are superposed:

$$
\left\{\begin{array}{l}
\sigma_{x(\mathrm{I}+\amalg)}=\sigma_{x \mathrm{I}}+\sigma_{x \amalg} \\
=-\frac{K_{\mathrm{I}}}{\sqrt{2 \pi r}} \cos \frac{\theta}{2}\left[1-\sin \frac{\theta}{2} \sin \frac{3 \theta}{2}\right]-\frac{K_{\amalg}}{\sqrt{2 \pi r}} \sin \frac{\theta}{2}\left[2+\cos \frac{\theta}{2} \cos \frac{3 \theta}{2}\right] \\
\sigma_{y(\mathrm{I}+\amalg)}=\sigma_{y \mathrm{I}}+\sigma_{y \amalg} \\
=-\frac{K_{\mathrm{I}}}{\sqrt{2 \pi r}} \cos \frac{\theta}{2}\left[1-\sin \frac{\theta}{2} \sin \frac{3 \theta}{2}\right]+\frac{K_{\amalg}}{\sqrt{2 \pi r}} \cos \frac{\theta}{2} \sin \frac{\theta}{2} \cos \frac{3 \theta}{2} \\
\tau_{x y(\mathrm{I}+\amalg)}=\tau_{x y \mathrm{I}}+\tau_{x y} \amalg \\
=-\frac{K_{\mathrm{I}}}{\sqrt{2 \pi r}} \cos \frac{\theta}{2} \sin \frac{\theta}{2} \cos \frac{\theta}{2}+\frac{K_{\amalg}}{\sqrt{2 \pi r}} \cos \frac{\theta}{2}\left[1-\sin \frac{\theta}{2} \sin \frac{3 \theta}{2}\right]
\end{array}\right.
$$

For the non through section, if theta is zero, the formula (3) is simplified as:

$$
\left\{\begin{array}{l}
\sigma_{x(\mathrm{I}+\amalg)}=\sigma_{x \mathrm{I}}+\sigma_{x \amalg}=-\frac{K_{\mathrm{I}}}{\sqrt{2 \pi r}} \\
\sigma_{y(\mathrm{I}+\amalg)}=\sigma_{y \mathrm{I}}+\sigma_{y \amalg}=-\frac{K_{\mathrm{I}}}{\sqrt{2 \pi r}} \\
\tau_{x y(\mathrm{I}+\amalg)}=\tau_{x y \mathrm{I}}+\tau_{x y \amalg}=\frac{K_{\amalg}}{\sqrt{2 \pi r}}
\end{array}\right.
$$

According to the stress superposition principle, the main structural plane stress field is through segment, change under normal linear surface stress distribution $\left(p_{w}\right)$ and non superposition effect through segment equivalent stress, fracture hydraulic stress assumption $\left(p_{w}\right)$ in triangular distribution (Figure 4).
Calculation of the hydraulic pressure $\left(p_{w}\right)$ under the action of the main control surface:

$$
p_{w}=\gamma_{w} h
$$

Type: $\gamma_{w}$ is the volume density of fissure water $\left(\mathrm{kN} / \mathrm{m}^{3}\right)$; The water filling height in the main control structure is $\mathrm{h}(\mathrm{m})$; The length of $1 / 3$ through the main control section of the structure surface under the natural state, and the $2 / 3$ length of the rainstorm state.

According to equation (5) fracture hydraulic calculations should be there is a big error between the testing value and theory value, the test values are generally lower than the theoretical value, in view of this, the 13 of the fissure water pressure model test, the reduction coefficient $\xi$, according to the test data obtained reduction coefficient $\xi$ formula:

$$
\xi=k_{1} a_{1}^{2}+k_{2} a_{1}+k_{3}
$$


Type: $a_{1}$ in the form of the main control structure, the average opening degree, generally take $0.2 \sim 2.0 \mathrm{~cm}$ between; $k_{1}, k_{2}, k_{3}$ respectively, take $-0.36,0.45,0.57$.

Using formula (6), the stress correction formula of dangerous rock fracture is established:

$$
p_{w}=\xi \gamma_{w} h
$$

According to the stress intensity factor Handbook [14], the intensity factor formula of the normal distribution stress PW of the main structure plane through the lower surface and linearly varying with the lower surface:

$$
K_{\mathrm{I} w}=0.68 p_{w} \sqrt{\pi h}
$$

The stress field at the crack tip of the dangerous rock can only be affected by the hydraulic pressure of the dam (8) by substituting (1):

$$
\left\{\begin{array}{l}
\sigma_{x}^{\prime}=\frac{K_{I w}}{\sqrt{2 \pi r}} \cos \frac{\theta}{2}\left[1-\sin \frac{\theta}{2} \sin \frac{3 \theta}{2}\right] \\
\sigma_{y}^{\prime}=\frac{K_{I w}}{\sqrt{2 \pi r}} \cos \frac{\theta}{2}\left[1+\sin \frac{\theta}{2} \sin \frac{3 \theta}{2}\right] \\
\sigma_{x y}^{\prime}=\frac{K_{I w}}{\sqrt{2 \pi r}} \cos \frac{\theta}{2} \sin \frac{\theta}{2} \cos \frac{3 \theta}{2}
\end{array}\right.
$$

When the non through section $(\theta)$ of the main control structure is zero, the formula (9) can be simplified as:

$\left\{\begin{array}{l}\sigma_{x}^{\prime}=\frac{K_{I w}}{\sqrt{2 \pi r}} \\ \sigma_{y}^{\prime}=\frac{K_{I w}}{\sqrt{2 \pi r}}\end{array}\right.$

Under load, the main structure face end point $\mathrm{O}$ with high stress concentration, there will be damage zone, by type (4) is obtained through cross section on stress field are different. This paper uses the method of equivalent stress distribution, stress assumption on the discontinuous sum and the total force and the same, using the equivalent stress distribution of force to replace the non through on the segment (Figure 4).

The equivalent stress field of the non penetrating section can be obtained by figure 4 :

$$
\left\{\begin{array}{l}
\overline{\sigma_{x}}=-\frac{\int_{0}^{\mathrm{a}_{0}} \sigma_{x(\mathrm{I}+\amalg)} d r}{\mathrm{a}_{0}}=-\frac{1}{\mathrm{a}_{0}} \int_{0}^{\mathrm{a}_{0}} \frac{K_{\mathrm{I}}}{\sqrt{2 \pi r}} d r=-\frac{2 K_{\mathrm{I}}}{\sqrt{2 \pi \mathrm{a}_{0}}} \\
\overline{\sigma_{y}}=-\frac{\int_{0}^{\mathrm{a}_{0}} \sigma_{y(\mathrm{I}+\amalg)} d r}{\mathrm{a}_{0}}=-\frac{1}{\mathrm{a}_{0}} \int_{0}^{\mathrm{a}_{0}} \frac{K_{\mathrm{I}}}{\sqrt{2 \pi r}} d r=-\frac{2 K_{\mathrm{I}}}{\sqrt{2 \pi \mathrm{a}_{0}}} \\
\overline{\tau_{x y}}=-\frac{\int_{0}^{\mathrm{a}_{0}} \tau_{x y(\mathrm{I}+\amalg)} d r}{\mathrm{a}_{0}}=\frac{1}{\mathrm{a}_{0}} \int_{0}^{\mathrm{a}_{0}} \frac{K_{\amalg}}{\sqrt{2 \pi r}} d r=\frac{2 K_{\amalg}}{\sqrt{2 \pi \mathrm{a}_{0}}}
\end{array}\right.
$$

By superposition of formula (11) and formula (10), the total equivalent stress field at the crack tip under the hydraulic pressure of the fracture at the through section is obtained: 


$$
\left\{\begin{array}{l}
\sigma_{\mathrm{x}\left(\mathrm{I}+\Pi+p_{w}\right)}=\overline{\sigma_{x}}+\sigma_{x}^{\prime}=-\frac{2 K_{\mathrm{I}}}{\sqrt{2 \pi \mathrm{a}_{0}}}+\frac{K_{\mathrm{I} w}}{\sqrt{2 \pi r}} \\
\sigma_{\mathrm{y}\left(\mathrm{I}+\Pi+p_{w}\right)}=\overline{\sigma_{y}}+\sigma_{y}^{\prime}=-\frac{2 K_{\mathrm{I}}}{\sqrt{2 \pi \mathrm{a}_{0}}}+\frac{K_{\mathrm{I} w}}{\sqrt{2 \pi r}} \\
\tau_{\mathrm{xy}\left(\mathrm{I}+\Pi+p_{w}\right)}=\overline{\tau_{x y}}+\tau_{x y}^{\prime}=\frac{2 K_{\Pi}}{\sqrt{2 \pi a_{0}}}
\end{array}\right.
$$

\section{THE FAILURE CRITERION OF INSTABILITY UNDER D-P THEORY IS ESTABLISHED}

This paper uses Drucker Prager (Drucker-Prager) criterion (D-P criterion). The criterion considers the role of hydrostatic pressure, overcomes the weakness of M-C standard numerical calculation theory has been difficult, rock mechanics at home and abroad and the finite element calculation is very big promotion.

The D-P criterion is extended and generalized on the basis of the Mises criterion and the C-M criterion in plastic mechanics:

$$
f=a I_{1}+\sqrt{J_{2}}-K=0
$$

In the formula, $I_{1}=\sigma_{1}+\sigma_{2}=\sigma_{x}+\sigma_{y}$ is the first stress invariant under the plane stress state;

$$
J_{2}=\frac{1}{6}\left[\left(\sigma_{1}-\sigma_{2}\right)^{2}+\sigma_{1}^{2}+\sigma_{2}^{2}\right]=\frac{1}{6}\left[\left(\sigma_{x}-\sigma_{y}\right)^{2}+\sigma_{x}^{2}+\sigma_{y}^{2}+6 \tau_{x y}^{2}\right] \text { is a }
$$

$$
\begin{aligned}
& a=\frac{2 \sin \varphi}{\sqrt{3}(3-\sin \varphi)} ; \\
& K=\frac{6 c \cos \varphi}{\sqrt{3}(3-\sin \varphi)} ;
\end{aligned}
$$

$c$ and $\varphi$ are the cohesion (MPa) and internal friction angle $\left(^{\circ}\right)$ of rock mass materials.

The total equivalent stress of formula (12) is replaced by (13) $\sigma_{x}, \sigma_{y}, \tau_{x y}$, tidy up:

$$
I_{1}=\frac{2 K_{\mathrm{I} w}}{\sqrt{2 \pi r}}-\frac{4 K_{\mathrm{I}}}{\sqrt{2 \pi \mathrm{a}_{0}}}
$$

$$
J_{2}=\frac{1}{3}\left(\frac{K_{\mathrm{I} w}}{\sqrt{2 \pi r}}-\frac{2 K_{\mathrm{I}}}{\sqrt{2 \pi \mathrm{a}_{0}}}\right)^{2}+\frac{2 K_{\Pi}^{2}}{\pi \mathrm{a}_{0}}
$$

Type (19), type (20) into the equation (13), tidy up: stress deviator second invariant;

$$
a\left(\frac{2 K_{\mathrm{I} w}}{\sqrt{2 \pi r}}-\frac{4 K_{\mathrm{I}}}{\sqrt{2 \pi \mathrm{a}_{0}}}\right)+\sqrt{\frac{1}{3}\left(\frac{K_{\mathrm{I} w}}{\sqrt{2 \pi r}}-\frac{2 K_{\mathrm{I}}}{\sqrt{2 \pi \mathrm{a}_{0}}}\right)^{2}+\frac{2 K_{\Pi}^{2}}{\pi \mathrm{a}_{0}}}-K=0
$$

When pure pressure is applied, $K_{\Pi}=0$, formula (21) can be reduced to:

$$
\left(2 a+\frac{1}{\sqrt{3}}\right)\left(\frac{K_{\mathrm{I} w}}{\sqrt{2 \pi r}}-\frac{2 K_{\mathrm{I}}}{\sqrt{2 \pi \mathrm{a}_{0}}}\right)-K=0
$$

In pure shear, $K_{\mathrm{I}}=0$, formula (21) can be reduced to:

$$
\frac{2 a K_{\mathrm{I} w}}{\sqrt{2 \pi r}}+\sqrt{\frac{1}{3} \frac{K_{\mathrm{I} w}^{2}}{\sqrt{2 \pi r}}+\frac{2 K_{\Pi}^{2}}{\pi \mathrm{a}_{0}}}-K=0
$$

In all the formulas, the values of $a$ and $K$ are obtained by laboratory tests. The cohesive force $\left({ }^{c}\right)$ and the internal friction angle $(\varphi)$ of rock mass are obtained; The length of the non penetrating section of the main control surface of the a0 is obtained by field measurements; $K_{\text {I }=} K_{\text {Ic }}$ and $K_{\Pi=} K_{\Pi c}$ fracture toughness of rock type I and type II for single side cracks are obtained by experiment; The fracture toughness of the $K_{\mathrm{I} w}=K_{\mathrm{I} w c}$ main control surface is only affected by the fracture water pressure, and is measured by the actual situation at the site. The failure criterion of unstable rock mass is established by using (16). The formula is simple and the parameters are easy to obtain, so it can be used as a criterion for the failure of the compression shear type dangerous rock. 


\section{CONCLUSION}

Firstly, based on the theory of linear elastic fracture mechanics, a fracture mechanics model of compression shear sliding dangerous rock body is established; By using stress equivalent method, the expressions of equivalent stress field of the main structure face are obtained.

Secondly, the Drucker-Prager (Drucker-Prager) criterion, failure criterion of compression shear sliding rock is established; Considering the two cases, the differential equations of the failure criterion of unstable rock under two conditions are obtained.

Thirdly, The criterion of failure to establish is based on the premise that the main control structure faces zero along the direction of theta, and there are some differences. Therefore, the established criteria need to be further studied and perfected to compensate for the lack of the criterion of instability failure.

\section{ACKNOWLEDGEMENTS}

This study in the paper was funded by The National Natural Science Foundation of China (No. 11272185, 51678097 and 50678182), and Chongqing University Innovation Team; The study Financially supported by the Program (201309) for Par-Eu Scholars in Chongjing and Chongqing Jiaotong University Innovation Fund (No. 20150101, CYB15110)

\section{REFERENCE}

[1] Chen H K, Xian X F, Tang H M. Stability analysis method for perilous rock by fracture mechanics 2009. Journal of Chongqing University, 32(4):434-437.

[2] Chen H K, Tang H M. Method to calculate fatigue fracture life of control fissure in perilous rock 2007. Applied Mathematics and Mechanics, 28(5): 575-580.

[3] Chen H K, Zhang R G, Tang H M, et al. Elastic \& impulsive dynamic parameters of a ruptured compression-shear perilous rock 2012. Journal of Vibration and Shock, 31(24):30-33.

[4] Wang L F, Chen H K, Tang H M. The aging calculate method for perilous rock stability reliability based on fracture mechanics and optimization method 2013. Journal of Wuhan University of Technology, 35(4): 434-437.

[5] Wu Y, He S M, Li X P, et al. Failure mechanism and diagnosis method of dangerous crack rock after earthquake 2010. Journal of Sichuan University (Engineering Science Edition), 42(5): 185-190.

[6] Li J H, Wu L Z. Calculation of stress intensity factor of unstable rock 2013. Journal of Engineering Geology, 21(2): 236-242.

[7] Liu C J, Zhang S F, Ding L Q, et al. Identification of dangerous rock mass of high slope and study of anchoring method based on laser scanning 2012. Chinese Journal of Rock Mechanics and Engineering, 31(10): 2139-2146.

[8] Yu M M, Jia Z X, Li X X. Analysis on the stability of the dangerous rock in high slope based on the mechanics model of cantilever 2012. Safety and Environmental Engineering, 19(1): 117-119.

[9] Zhang Y X, Lu L, Zhang S P, et al. Development and failure principle of differential weathering overhanging rock 2010. Journal of Civil, Architectural \& Environmental Engineering, 2010, 32(2): 1-6.

[10] Zheng A X, Luo X Q, Shen H. Numerical simulation and analysis of deformation and failure of jointed rock slopes by extended finite element method 2013. Rock and Soil Mechanics, 34(8): 2371-2377.

[11] Shi Y C, Qiu R D, Sun J J, et al. Analysis of dynamic response of dangerous rock mass reinforced by prestressed anchor cables under seismic loads 2011. Rock and Soil Mechanics, 32(4):1157-1162.

[12] Chen H K, Tang H M, Wang L F, et al. Theory and application of collapse evolution of perilous rock 2009. Beijing: Science Press.

[13] Tang H M, Chen H K. Revised method of water pressure in control fissure of perilous rock mass 2008. The Chinese Journal of Geological Hazard and control, 19(4):86-90

[14] Institute of space research China. Handbook of stress intensity factors 1981. Beijing: Science Press.

[15] Deng C J, He G J, Zheng Y R. Studies on Drucker-Prager yield criterions based on M-C yield criterion and application in geotechnical engineering 2006. Chinese Journal of Geotechnica Engineering, 28(6):735-739. 